\title{
Spontaneous Chondrocyte Maturation on 3D-Chitosan Scaffolds
}

\section{Serafim M Oliveira ${ }^{1,2,3}$, Gloria Turner ${ }^{1}$, Simone P Rodrigues ${ }^{2,3}$, Mário A Barbosa ${ }^{3}$, Mani Alikhani ${ }^{1,4}$ and Cristina C Teixeira ${ }^{1,4,5 *}$}

${ }^{1}$ Consortium for Translational Orthodontic Research, New York University College of Dentistry, New York, USA

${ }^{2}$ Department of Mechanical Engineering, ESTV-Escola Superior de Tecnologia de Viseu, Viseu, Portugal

${ }^{3}$ INEB-Instituto de Engenharia Biomédica, Porto, Portugal

${ }^{4}$ Department of Orthodontics, New York University, College of Dentistry, New York, NY, USA

${ }^{5}$ Department of Basic Science and Craniofacial Biology, New York University College of Dentistry, New York, USA

\begin{abstract}
The endochondral ossification mechanism provides promising new insight into potential techniques for repairing bone defects. The methods for replicating endochondral ossification in culture, although diverse, have yet to identify a procedure that reflects natural conditions, while satisfying the practical, experimental needs for efficiency and maneuverability. In this study, we present a novel approach that addresses all of these concerns. Coordinated proliferation and differentiation of chondrocytes in a transient cartilage is required for endochondral bone growth, but the mechanisms and pathways that control these processes are not completely understood. For lack of alternative methods, chondrocyte cultures are commonly pushed to differentiation with growth factors and supplements, like retinoic acid. We have developed a tissue culture model, using a 3D-chitosan sponge, which supports the proliferation and differentiation of chondrocytes, without exposure to supplements, and which matures into a near replica of the growth plate.
\end{abstract}

Keywords: Chondrocytes maturation; Chitosan; Alkaline phosphatase; Tissue engineering; Endochondral ossification

\section{Introduction}

In the last few decades, it has become standard practice to repair bone defects by filling the gaps of skeletal contours with biomaterials that serve as the scaffold for regenerating tissue. Our previous work has demonstrated that chondrocytes from transient cartilage, similar to growth plate cells, can be seeded onto a scaffold, and develop into bone after implantation into nude mice $[1,2]$. From these successes, it is clear that further research is necessary to understand chondrocytes and their potential role in bone tissue engineering. In this study, we present a novel approach to culturing transient cartilage as a strategy for studying chondrocyte maturation. Chondrocyte maturation is critical during endochondral ossification, which is one of two natural processes that lead to skeletal formation. The second pathway is intramembranous ossification, in which mesenchymal cells differentiate directly into osteoblasts, without an intermediate cartilage stage. During endochondral ossification, a cartilage model is partially replaced by bone. The growth plate, a disc of cartilage located between the epiphyseal and metaphyseal osteogenic centers, is responsible for pre-natal and postnatal growth of long bones. Chondrocytes in the growth plates undergo rapid division, followed by maturation and death by apoptosis. There are well documented changes in the phenotype of chondrocytes during maturation, including increased alkaline phosphatase activity [3,4], elevated synthesis of type $\mathrm{X}$ collagen and matrix metalloproteinase 13 (MMP13) [5-8], and down regulation of type II collagen production [9], synthesis of extracellular matrix [10] and mineralization [11-13]. These changes lead to formation of an unvascularized, calcified, cartilage structure, in which chondrocytes survive hypoxia and a low supply of nutrients. This structure is soon invaded by marrow constituents and capillary buds. The growth plate cartilage is gradually replaced by bone, as osteoclasts reabsorb the calcified matrix and osteoblasts deposit bone. These facts make the endochondral mechanism most suitable for a tissue culture environment and efficient bone engineering.

In question now are the various techniques by which one can further study the proliferation of chondrocytes and their subsequent maturation. Dynamic cultures (with the use of spinner flask, rotating wall bioreactors and perfusion systems) which intend to simulate natural conditions, have shown variable success, and been commercially developed [14-18]. However, it remains most common to culture skeletal tissue under static conditions (monolayer, 3D-micromass, 3D-scaffolds or organ culture), which can be manipulated more efficiently (Figure 1 summarizes in vitro approaches). Chondrocytes cannot induce bone formation unless they undergo maturation, and release the growth factors needed to induce vascularization, osteoblasts and osteoclast activity. Most researchers studying chondrocyte differentiation have used retinoic acid (RA) to accelerate maturation [19-24]. While most studies assume these supplements are necessary, our current data shows that the scaffold material alone can direct cell behavior and differentiation. Here, we report a static culture model in which a 3D-chitosan scaffold material was sufficient to induce chondrocyte proliferation and maturation.

We studied the behavior of chondrocytes in 3D-chitosan sponges, without an inducer of maturation. Important signs of chondrocyte maturation (matrix synthesis, increased activity of alkaline phosphatase (ALP), expression of type X collagen, and decrease of expression of type II collagen) were detected. These results strongly suggest that 3Dchitosan sponges create a microenvironment that favors, and is sufficient for chondrocyte maturation to occur in a way that mimics their development in the growth plate.

*Corresponding author: Cristina C Teixeira, DMD, MS, PhD, Department of Basic Science and Craniofacial Biology, New York University College of Dentistry, 345 East 24th Street, New York, NY 10010, USA, Tel: +(212) 9989958; E-mail: cristina.teixeira@nyu.edu

Received January 03, 2013; Accepted February 12, 2013; Published February 15,2013

Citation: Oliveira SM, Turner G, Rodrigues SP, Barbosa MA, Alikhani M, et al (2013) Spontaneous Chondrocyte Maturation on 3D-Chitosan Scaffolds. J Tissue Sci Eng 4: 124. doi:10.4172/2157-7552.1000124

Copyright: (c) 2013 Oliveira SM, et al. This is an open-access article distributed under the terms of the Creative Commons Attribution License, which permits unrestricted use, distribution, and reproduction in any medium, provided the original author and source are credited. 


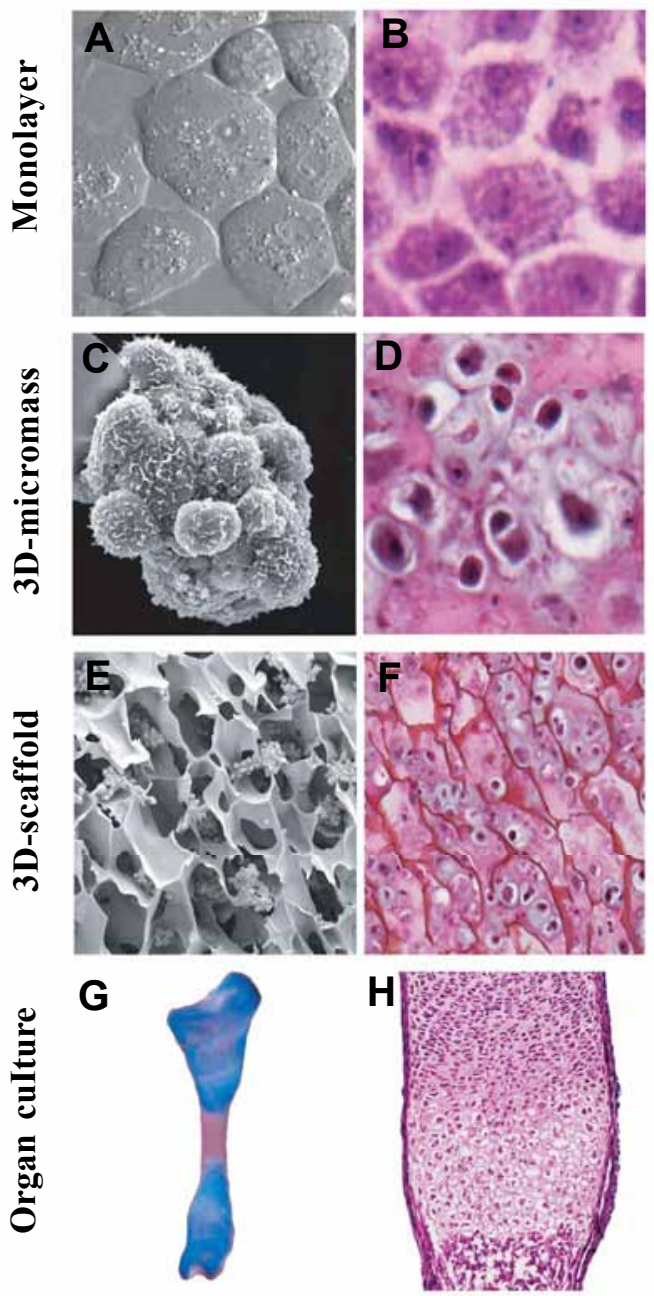

Figure 1: In vitro and ex vivo models to study chondrocyte maturation.

Chondrocyte maturation is critical during endochondral ossification. Different culture approaches have been utilized to investigate the chondrocyte phenotypical changes during maturation, as summarized here. Monolayer chondrocytes with a flat and polygonal morphology seen by DIC light microscopy (A) and H\&E staining (B). 3-D micromass cultures seen by SEM (C) and H\&E staining (D). Chondrocytes cultures in chitosan sponges, seen by SEM $(E)$ and $H \& E$ staining $(F)$. Ex vivo, organ culture of embryonic tibia stained with alcian blue and alizarin red $(G)$ and $H \& E(H)$.

\section{Materials and Methods}

\section{Chitosan scaffold preparation}

Squid pen chitosan (chitosan 123), supplied by France Chitine, was initially purified with a degree of acetylation (DA) $\cong 4 \%$, and subsequently prepared by heterogeneous de-acetylation, as described by Amaral et al. [24]. The physicochemical properties of the resultant chitosan have been published elsewhere by Oliveira et al. [1].Threedimensional (3D) porous scaffolds were prepared from $2 \%(\mathrm{w} / \mathrm{v})$ chitosan acidic solutions via thermally induced phase separation, and subsequent sublimation of the ice crystals. The chitosan solution was transferred to 24 -well polystyrene-coated tissue culture plates $(800 \mu \mathrm{L} /$ well), kept overnight at $80^{\circ} \mathrm{C}$, and freeze dried (Virtis Freezemobile$12 \mathrm{EL})$, to obtain a porous structure. The resultant sponges were cut into
$4 \times 4 \times 1 \mathrm{~mm}^{3}$ pieces, immersed in $100 \%$ ethanol, and then re-hydrated in serial dilutions of ethanol. The pieces were finally immersed in Dulbecco's Modified Eagle Medium (DMEM) (Cellgro, Mediatech Inc.) for two 20-minute intervals.

\section{Chondrocytes culture}

Cephalic (CP) and caudal (CD) chondrocytes were isolated from the sterna of 14-day old chicken embryos, according to the method described by Iwamoto et al. [23]. CP chondrocytes from the upper region of the sternum have the potential to undergo maturation and induce bone formation. CD chondrocytes from the lower region of the sternum behave as permanent cartilage cells, and were therefore, used as controls. Freshly isolated chondrocytes were plated in $100 \mathrm{~mm}$ tissue culture dishes and allowed to proliferate at $37^{\circ} \mathrm{C}$ and $5 \% \mathrm{CO}_{2}$ in $10 \mathrm{ml}$ complete medium (DMEM containing 10\% Nu serum (Fisher scientific, Fairlawn, NJ), 2 mM L-glutamine, and $100 \mathrm{U} / \mathrm{ml}$ penicillin/ streptomycin (Sigma-Aldrich, St Louis, MO)). After one week of proliferation, chondrocytes were designated for either monolayer or 3D-scaffold studies. For monolayer, chondrocytes were re-plated onto a 12-well plate (2,00,000 chondrocytes/well), and cultured for an additional 10 days. During the last 5 days, the wells were treated with $\mathrm{RA}$ at different concentrations $(0 \mathrm{nM}, 10 \mathrm{nM}, 35 \mathrm{nM}$ and $100 \mathrm{nM})$. For 3D-scaffold cultures, 2,00,000 chondrocytes in a $15 \mu \mathrm{L}$ suspension were seeded onto a single hydrated, chitosan sponge in each well of a 96-well plate, and incubated 2 hours. $200 \mu \mathrm{l}$ of complete medium was added to each well and the plates were cultured for an additional 10 days. Specimens from both culture types were then used for testing and imaging. In addition, growth plates from 19 day chick embryos were collected, and the proliferative and hypertrophic regions separated and analyzed.

\section{Immunohistochemistry and imaging}

For immunohistochemistry, samples were embedded in paraffin and cut into $5 \mu \mathrm{m}$ sections. The paraffin was removed and the samples were re-hydrated. Immunostaining was performed using a Vectastain $\mathrm{ABC}$ kit (Vector Laboratories, Burlingame, CA), and antibodies against chick alkaline phosphatase. Sections were counterstained with Alcian blue ( $1 \%$ alcian blue in $3 \%$ acetic acid), and sponge samples were additionally stained with light green, then mounted and scanned on Scan Scope GL optical microscope (Aperio, Bristol, UK). Scanning electron microscopy (SEM) samples were washed with phosphate buffered saline, fixed ( $2 \%$ gluteraldehyde in $0.1 \mathrm{M}$ sodium cocodylate $\mathrm{HCl}$ buffer with $0.1 \mathrm{M}$ sucrose, $\mathrm{pH} 7.2$ ) overnight at $4^{\circ} \mathrm{C}$, dehydrated in serial ethanol solutions at room temperature, and critical point dried (Denton Vaccum, Cherry Hill, NJ). Samples were cut, mounted on steel stubs, sputter coated with gold palladium (Emitech K-650), and analyzed by SEM (Hitachi S3500 N, Schaumburg, IL).

\section{Cell solubilization}

Three sets of three sponges for both CP and CD chondrocytes were washed with phosphate buffer solution, then immersed in $150 \mu \mathrm{l} 0.1 \%$ Triton-X (Fisher Scientific), and homogenized in eppendorf tubes on ice for 1 hour. Scaffolds were then crushed manually and centrifuged at $3000 \mathrm{rpm}$ for 2 minutes. Supernatant was collected and used for DNA quantification and ALP analysis.

\section{DNA quantification and alkaline phosphatase staining and activity}

Total DNA amount was measured according to the procedure described by Teixeira et al. [25], using a bisBenzididazole dye (Hoechst 
33258 dye; Polyscience, Northampton, UK). Fluorescence was measured at $365 \mathrm{~nm}$ excitation and $460 \mathrm{~nm}$ emission wavelengths. The results were extrapolated from a standard curve using salmon testis DNA (Sigma-Aldrich). Fluorescence was measured by spectrofluorometer (Beckman Coulter DU-640), at wavelengths of 365 and $460 \mathrm{~nm}$. A standard curve, using calf thymus DNA (Sigma) and regression analysis, was used to calculate DNA amount. ALP staining in monolayer and activity measurements was performed as described by Iwamoto et al. [23] and Leboy et al. [26], with the use of colorimetric assays. ALP activity was expressed as nmol of product $/ \mathrm{min} / \mu \mathrm{g}$ of DNA to correct for cell number differences; 1 absorbance unit change corresponds to $64 \mathrm{nmol}$ product.

\section{RNA preparation and analysis}

Chondrocyte mRNA was collected for use in real time RT-PCR. RNA was isolated from cells cultured in $3 \mathrm{D}$-scaffolds at day 5 and 10 , and from tibia growth plate, as described previously [1]. Real-time RT-PCR measurements were performed using QuantiTect SYBR Green RT-PCR kit (Qiagen Inc.), a DNA Engine Optican2 system (Roche Molecular Systems, Pleasanton, CA), and primers specific for chick genes. as previously described [1]. Relative transcript levels are presented as a fold change in gene expression and calculated using the threshold cycle $(\mathrm{Ct})$ and following formula, where "CD" refers to $\mathrm{CD}$ chondrocytes, "CP" refers to $\mathrm{CP}$ chondrocytes, and "RP" refers to the acidic ribosomal protein: $\mathrm{x}=2^{\Delta \Delta \mathrm{Ct}}$, in which $\Delta \Delta \mathrm{Ct}=\Delta \mathrm{E}-\Delta \mathrm{C}$, and $\Delta \mathrm{E}=\mathrm{CtCP}-\mathrm{CtRP}$, and $\Delta \mathrm{C}=\mathrm{CtCD}-\mathrm{CtRP}$. A $\Delta \Delta \mathrm{Ct}<0$ was considered to be an increase in gene expression, while a $\Delta \Delta \mathrm{Ct}>0$ was considered to be a decrease.

\section{Statistical analysis}

All experiments were repeated either 3 or 4 times, and the mean and standard deviation of the results were determined. Significant differences between test groups and controls were assessed by ANOVA. Significance was set at $\mathrm{p}<0.05$.

\section{Results}

\section{Monolayer culture staining}

We choose a well-described culture model, extensively used in studies of chondrocyte maturation. CP chondrocytes grown in monolayer for 1 week undergo maturation in response to RA treatment. ALP activity visualized by the red/pink staining (Figure 2 ), increases only in CP chondrocytes, in a dose dependent manner. However, CP chondrocytes in monolayer culture, in the absence of RA, do not undergo maturation. CD chondrocytes do not respond to RA treatment, and are therefore, used as controls, representing a stable cartilage phenotype.

\section{Chondrocyte proliferation and matrix synthesis on chitosan sponge}

To evaluate the extent of proliferation and maturation of chondrocytes in the chitosan sponge; in the absence of RA as maturation inducer, we examined the morphology and histology of these cells. Images from SEM show that the chitosan sponge itself was homogenous, both in the size and distribution of its pores (Figure 3A). Ten days after $\mathrm{CD}$ and $\mathrm{CP}$ chondrocytes were seeded onto the sponges, they proliferated and formed an abundant extracellular matrix, completely filling the pores of the sponges, as seen in SEM images (Figure 3B-D) and H\&E stained sections (Figure 3E-G). Alcian blue staining (Figure $3 \mathrm{H}$ and $\mathrm{I}$ ) shows the high proteoglycan content in the extracellular matrix generated by both $\mathrm{CP}$ and $\mathrm{CD}$ chondrocytes.

\section{Chondrocyte maturation on chitosan sponges}

While histological appearance for both $\mathrm{CD}$ and $\mathrm{CP}$ chondrocytes are comparable (Figure 3), with no significant morphological differences between the two cell types, the phenotype of these cells is very different. Analysis of ALP activity, an important marker of hypertrophy, shows that $\mathrm{CP}$ chondrocytes present significantly higher levels of enzymatic activity than CD cells $(\mathrm{p}<0.05)$. More strikingly, we observe a time dependent increase in ALP activity in CP, even in the absence of RA treatment (Figure 4), representing a 6-fold increase in activity. Already by day five, ALP measurements in CP chondrocytes were statistically higher than those of $\mathrm{CD}$ chondrocytes, by a factor of

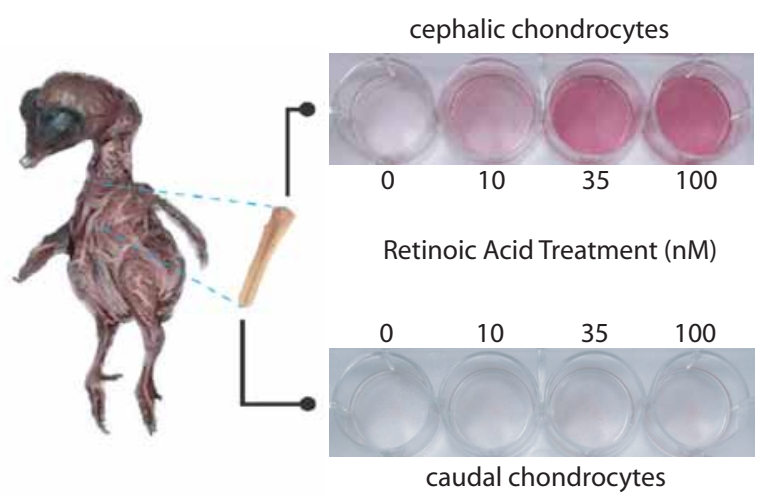

Figure 2: Culture model using embryonic chick chondrocytes.

Chondrocytes were removed from the cephalic and caudal region of 14 day chick embryos, cultured in monolayer and treated with retinoic acid (RA, 10, 35 , and $100 \mathrm{nM}$ ) to induce maturation. Photomicrographs of cells stained for alkaline phosphatase, show a dose dependent increase in this maturation marker (see increase in red color), only on cephalic chondrocytes.

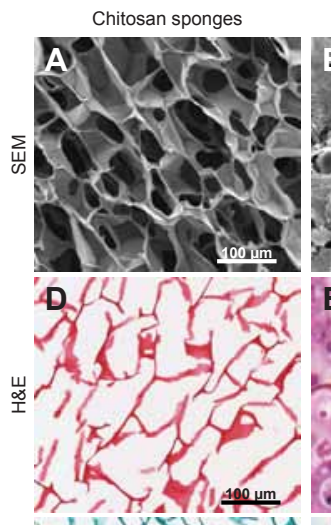

Cephalic chondrocytes
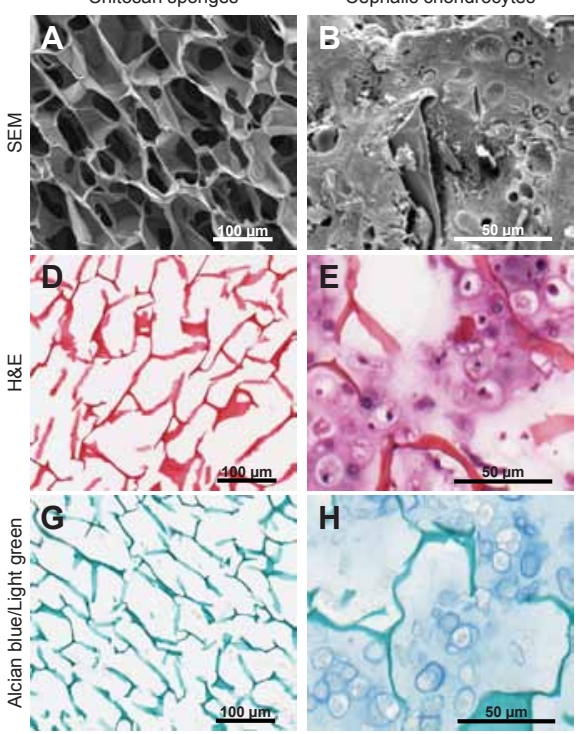

Caudal chondrocytes

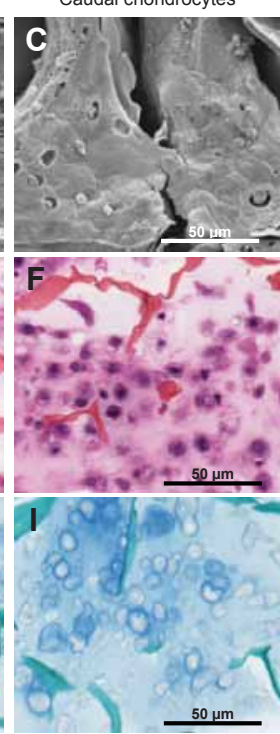

Figure 3: SEM and histological analysis of chondrocytes cultured in chitosan sponges.

Histological cross sections of sponges collected after 10 days in culture were visualized by SEM $(A, B, C), H \& E(D, E, F)$, and alcian blue to visualize proteoglycans in the matrix $(\mathrm{G}, \mathrm{H}, \mathrm{I})$. Some hypertrophic chondrocytes can be identified in the cultures of CP cells (compare $E$ to $F$ ). 
4. This difference increased by day 10 to a factor of 24 . ALP activity in CD cells did not increase, even when the culture period was extended to 15 days (data not shown).

\section{Chondrocytes in 3-D chitosan scaffolds mimic growth plate cell behavior}

$\mathrm{CP}$ and $\mathrm{CD}$ chondrocytes cultured in chitosan sponges for 10 days undergo rapid proliferation and matrix synthesis and maturation. Immuhistochemistry, SEM images and quantitative RTPCR results suggest that the cultured chondrocytes develop morphology, protein and gene expression profile, similar to growth plate chondrocytes. In SEM images (Figure 5, first column), CD and CP chondrocytes, after 10 days in culture on a 3D chitosan sponge, show morphology similar to cells from the proliferative and hypertrophic areas of the growth plate, respectively. Likewise, the level of ALP activity is comparable

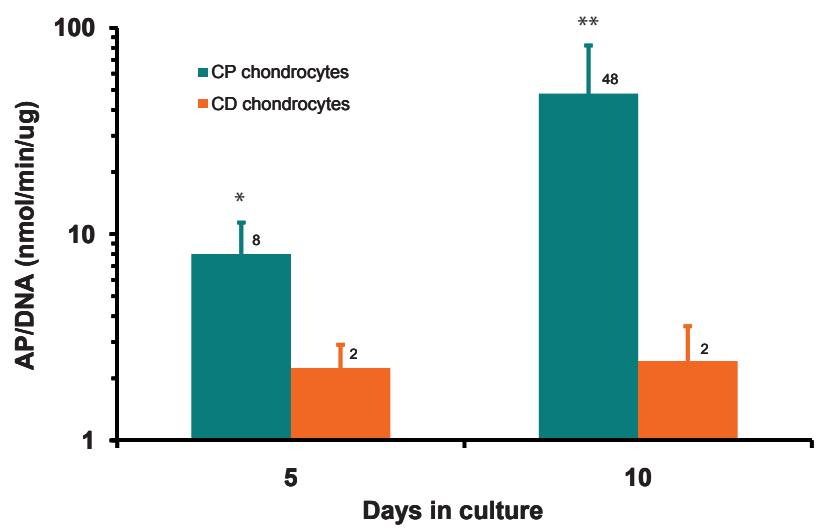

Figure 4: Alkaline phosphatase activity increases in CP chondrocytes culture in chitosan sponges.

$\mathrm{CP}$ and $\mathrm{CD}$ chondrocytes were cultured in chitosan sponges for 5 and 10 days, ALP activity was measured spectrophotometricaly, and normalized to the total DNA content of each sample. ALP activity exponentially increased in $\mathrm{CP}$ chondrocytes, while in $\mathrm{CD}$ chondrocytes, no significant changes where observed. "Significant difference from CP chondrocytes at day 5. " Significant difference from CD chondrocytes day 10.

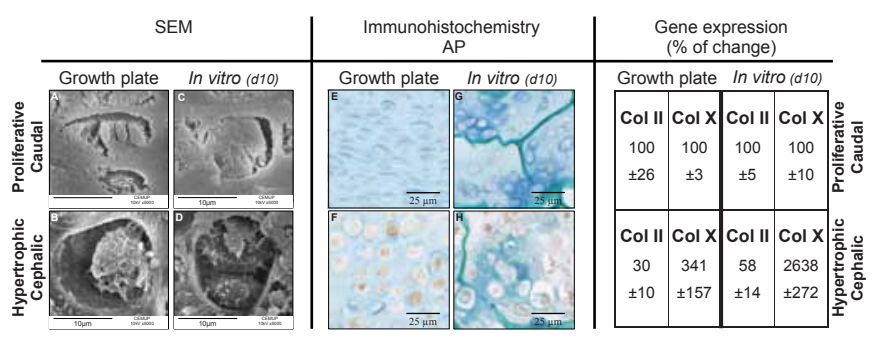

Figure 5: Comparison of phenotypical changes of chondrocytes in vivo and cultured in chitosan sponges.

$\mathrm{CP}$ and $\mathrm{CD}$ chondrocytes were cultured for 10 days in chitosan sponges, and samples collected for SEM, ALP immunostaining and Real time RT-PCR, using primers specific for chick genes. 19 day chick embryo tibia growth plates were analyzed in the same manner. Note the similar morphology of chondrocytes in the growth plate, and in the chitosan. Positive ALP staining is present in hypertrophic chondrocytes in the growth plate and CP cells in culture. Gene expression profiles are also similar in hypertrophic cells in the growth plate and the CP cells in culture. Expression levels are presented as "fold change" in mRNA levels in CP chondrocytes, when compared to CD chondrocytes or hypertrophic chondrocytes compare to proliferative. All values were significantly different. in cultured CP and CD cells to growth plate cells, as demonstrated by consistent patterns of staining for ALP (Figure 5, second column). The gene expression levels also follow a similar pattern (Figure 5, third column). As expected, expression of type II collagen decreased from the proliferative to the hypertrophic area of the growth plate. Similarly, levels of type II collagen were lower in maturing CP chondrocytes than immature $\mathrm{CD}$ chondrocytes, while Type $\mathrm{X}$ collagen expression increased with maturation, both in the growth plate cells and $\mathrm{CP}$ chondrocytes, in the chitosan sponges.

\section{Discussion}

Our in vitro studies demonstrate that a 3D-chitosan sponge, in the absence of inducers of maturation, can support chondrocyte proliferation and hypertrophy, as characterized by expression of type $\mathrm{X}$ collagen and high ALP activity. As it matures, the culture displays a behavior closely resembling that of the growth plate, making it a suitable model for researching the endochondral maturation process. Moreover, we found this model to be superior to other current techniques for reasons described below.

Several methods of culturing cells in static conditions have been used, as shown in figure 1. Culturing cells in a monolayer, either at low or high density, is the most accepted method. However, as shown in figure 2, when cultured in a monolayer, chondrocyte cells will only differentiate in the presence of RA or growth factors, and in some studies, they have been found to de-differentiate under prolonged culture conditions [27]. Most importantly, however, is the fact that monolayer cultures of any kind poorly reference the architecture of their native tissue. Micromass cultures are the ones that closely resemble chondrocyte differentiation and maturation, however, requiring weeks for terminal differentiation to occur and small pieces of tissue are obtained [28]

Finally, there is the method of organ culture, which yields a tissue closely representing the natural conditions of the cells. Mastering the technique, however, is prohibitively difficult and time consuming. Nor is it an ideal model for experiments requiring genetic manipulations, as it can be challenging to silence and over-express genes consistently throughout the tissue. Furthermore, organ cultures leave the researcher with little control over the profile of cell types in the developing tissue.

We are excited to report that cultures seeded onto a 3D-chitosan sponge display all the benefits of the above techniques, with none of the setbacks. In all of these previous works, cells were pushed into differentiation, using at least one of the following inducers: growth factors, co-culture or synthetic chemicals. However, in our current study, CP chondrocytes matured and proliferated without inducer application, into a single-cell morphology and multi-cell architecture, closely resembling that of the growth plate. We further observed that cephalic chondrocytes cultured on 3D-chitosan scaffolds are similar to chondrocytes from the hypertrophic area of growth plate, while caudal chondrocytes look more like chondrocytes from the proliferative area. Morphologically, cephalic chondrocytes grew in size, resembling hypertrophic chondrocytes. In addition to the morphology, ALP staining patterns and the trends of type X and type II collagen expression are similar in both cephalic chondrocytes, and those found in the hypertrophic area. A remarkable parallelism was detected between caudal chondrocytes cultured in 3D-chitosan sponges and chondrocytes harvested from the proliferative area of growth plate.

In previous studies, we have shown that such a transient cartilage 
template carries all the signals necessary to induce new bone formation, when implanted into the subcutaneous region of nude mice $[1,2]$. In light of these achievements, we consider the endochondral ossification mechanism to be a promising approach to tissue engineering and bone repair. We further argue that culturing chondrocytes on a 3D-chitosan sponge is the optimal method for studying the differentiation of chondrocytes and the induction of a transient cartilage phenotype.

\section{References}

1. Oliveira SM, Amaral IF, Barbosa MA, Teixeira CC (2009) Engineering endochondral bone: in vitro studies. Tissue Eng Part A 15: 625-634.

2. Oliveira SM, Mijares DQ, Turner G, Amaral IF, Barbosa MA, et al. (2009) Engineering endochondral bone: in vivo studies. Tissue Eng Part A 15: 635-

3. Lewinson D, Toister Z, Silbermann M (1982) Quantitative and distributional changes in the activity of alkaline phosphatase during the maturation of cartilage. J Histochem Cytochem 30: 261-269.

4. Pfander D, Swoboda B, Kirsch T (2001) Expression of early and late differentiation markers (proliferating cell nuclear antigen, syndecan-3, annexin $\mathrm{VI}$, and alkaline phosphatase) by human osteoarthritic chondrocytes. Am $\mathrm{J}$ Pathol 159: 1777-1783.

5. D'Angelo M, Billings PC, Pacifici M, Leboy PS, Kirsch T (2001) Authentic matrix vesicles contain active metalloproteases (MMP). a role for matrix vesicleassociated MMP-13 in activation of transforming growth factor-beta. J Bio Chem 276: 11347-11353.

6. D'Angelo M, Yan Z, Nooreyazdan M, Pacifici M, Sarment DS, et al. (2000) MMP-13 is induced during chondrocyte hypertrophy. J Cell Biochem 77: 678693.

7. Shen G (2005) The role of type X collagen in facilitating and regulating endochondral ossification of articular cartilage. Orthod Craniofac Res 8: 11-17.

8. Kirsch T, Harrison G, Golub EE, Nah HD (2000) The roles of annexins and types II and X collagen in matrix vesicle-mediated mineralization of growth plate cartilage. J Biol Chem 275: 35577-35583.

9. Mwale F, Billinghurst C, Wu W, Alini M, Webber C, et al. (2000) Selective assembly and remodelling of collagens II and IX associated with expression of the chondrocyte hypertrophic phenotype. Dev Dyn 218: 648-662.

10. Hunziker EB (1994) Mechanism of longitudinal bone growth and its regulation by growth plate chondrocytes. Microsc Res Tech 28: 505-519.

11. Anderson HC (1969) Vesicles associated with calcification in the matrix of epiphyseal cartilage. J Cell Biol 41: 59-72.

12. Anderson HC (1995) Molecular biology of matrix vesicles. Clin Orthop Relat Res 266-280.

13. Bonucci E (1967) Fine structure of early cartilage calcification. J Ultrastruct Res 20: 33-50.
14. Yu X, Botchwey EA, Levine EM, Pollack SR, Laurencin CT (2004) Bioreactorbased bone tissue engineering: the influence of dynamic flow on osteoblas phenotypic expression and matrix mineralization. Proc Natl Acad Sci U S A 101: 11203-11208.

15. Janssen FW, Oostra J, Oorschot Av, van Blitterswijk CA (2006) A perfusion bioreactor system capable of producing clinically relevant volumes of tissueengineered bone: in vivo bone formation showing proof of concept. Biomaterials 27: 315-323.

16. Ohyabu Y, Tanaka J, Ikada Y, Uemura T (2009) Cartilage tissue regeneration from bone marrow cells by RWV bioreactor using collagen sponge scaffold. Mater Sci Eng C 29: 1150-1155.

17. Lawrence BJ, Devarapalli M, Madihally SV (2009) Flow dynamics in bioreactors containing tissue engineering scaffolds. Biotechnol Bioeng 102: 935-947.

18. Stevens MM, Marini RP, Schaefer D, Aronson J, Langer R, et al. (2005) In vivo engineering of organs: the bone bioreactor. Proc Natl Acad Sci U S A 102 11450-11455.

19. De Luca F, Uyeda JA, Mericq V, Mancilla EE, Yanovski JA, et al. (2000) Retinoic acid is a potent regulator of growth plate chondrogenesis. Endocrinology 141 346-353.

20. Cowan CM, Aalami OO, Shi YY, Chou YF, Mari C, et al. (2005) Bone morphogenetic protein 2 and retinoic acid accelerate in vivo bone formation, osteoclast recruitment, and bone turnover. Tissue Eng 11: 645-658.

21. Li X, Schwarz EM, Zuscik MJ, Rosier RN, Ionescu AM, et al. (2003) Retinoic acid stimulates chondrocyte differentiation and enhances bone morphogenetic protein effects through induction of Smad1 and Smad5. Endocrinology 144 2514-2523.

22. Wang W, Kirsch T (2002) Retinoic acid stimulates annexin-mediated growth plate chondrocyte mineralization. J Cell Biol 157: 1061-1069.

23. Iwamoto M, Shapiro IM, Yagami K, Boskey AL, Leboy PS, et al. (1993) Retinoic acid induces rapid mineralization and expression of mineralization-related genes in chondrocytes. Exp Cell Res 207: 413-420.

24. Amaral IF, Sampaio P, Barbosa MA (2006) Three-dimensional culture of human osteoblastic cells in chitosan sponges: the effect of the degree of acetylation. $J$ Biomed Mater Res A 76: 335-346.

25. Teixeira CC, Hatori M, Leboy PS, Pacifici M, Shapiro IM (1995) A rapid and ultrasensitive method for measurement of DNA, calcium and protein content, and alkaline phosphatase activity of chondrocyte cultures. Calcif Tissue Int 56 252-256.

26. Leboy PS, Vaias L, Uschmann B, Golub E, Adams SL, et al. (1989) Ascorbic acid induces alkaline phosphatase, type $X$ collagen, and calcium deposition in cultured chick chondrocytes. J Biol Chem 264: 17281-17286.

27. Descalzi Cancedda F, Gentili C, Manduca P, Cancedda R (1992) Hypertrophic chondrocytes undergo further differentiation in culture. J Cell Biol 117: 427-435.

28. Zhang L, Su P, Xu C, Yang J, Yu W, et al. (2010) Chondrogenic differentiation of human mesenchymal stem cells: a comparison between micromass and pellet culture systems. Biotechnol Lett 32: 1339-1346. 